\title{
El análisis de red en la investigación clínica de la patogenia comórbida del COVID-19
}

\section{Network analysis in the clinical investigation of the comorbid pathogenesis of COVID.19}

\author{
Cristian Antony Ramos-Vera,a
}

\section{$\mathrm{DOI}$}

https://doi.org/10.35434/rcmhnaaa.2021.142.1092

\section{Señor editor:}

Un estudio publicado en la presente revista ha destacado el impacto de la infección provocada por la COVID-19 y un mayor riesgo de mortalidad en pacientes con diabetes mellitus (DM) e hipertensión arterial $(\mathrm{HA})^{(1)}$, lo cual es incluso más grave en aquellas personas con ambas condiciones comórbidas. Estos pacientes presentan mayores niveles de enzima convertidora de angiotensina 2 (ECA2) que interactúa con los componentes de la enfermedad de COVID-19 lo cual afianza un mayor riesgo de un síndrome de dificultad respiratoria aguda.

Es importante evaluar las asociaciones dinámicas entre tales estados de salud mediante la interacción de sus mecanismos fisiopatológicos para explorar la relación de la ECA2 con los biomarcadores inflamatorios que afectan la inmunidad adaptativa ${ }^{(2,3)}$ y otros mecanismos patogénicos potenciales que conducen a una mayor prevalencia de mortalidad cardiovascular y por cualquier otra causa ${ }^{(3,4)}$.

La investigación clínica ante la COVID-19 plantea desafíos específicos dado su estado crítico de comorbilidad con otras enfermedades crónicas de salud física y mental cuya evaluación requiere de una compleja interacción multifactorial etiológica, esto urge análisis más robustos que incluyan simultáneamente a todas las variables de estudio más allá del uso de métodos univariados en las ciencias de la salud, dado que estos modelos estadísticos no permiten explicar el fenómeno global investigado puesto que captan la acción aislada de cada variable respecto al resultado analizado.

Por lo tanto, esta carta presenta el modelo de red de estimaciones estadísticas (dirigidas o no dirigidas) compuesto por relaciones totales o parciales regularizadas (eliminación de las relaciones más espurias) que explora los vínculos que ocurren simultáneamente entre múltiples variables, representados por nodos que conforman el modelo dinámico ${ }^{(5,6)}$. Su representación gráfica facilita la interpretación de manera sencilla: mientras más gruesa sea la conexión entre las variables, mayor será la relación estadística.

Este método es esencial para avanzar en la investigación de la comorbilidad de COVID-19 mediante la evaluación de múltiples mecanismos biológicos, e incluir factores predisponentes y precipitantes. Varios estudios de esta nueva variante de red presentan variables de diversa medición clínica como factores genéticos o sustancias moleculares ${ }^{(7)}$, medidas fisiológicas y antropométricas ${ }^{(8)}$, condiciones crónicas $^{(8)}$, aspectos psicológicos ${ }^{(9)}$ y neuroanatómicos ${ }^{(10)}$ que permiten esclarecer la complejidad de los estados de

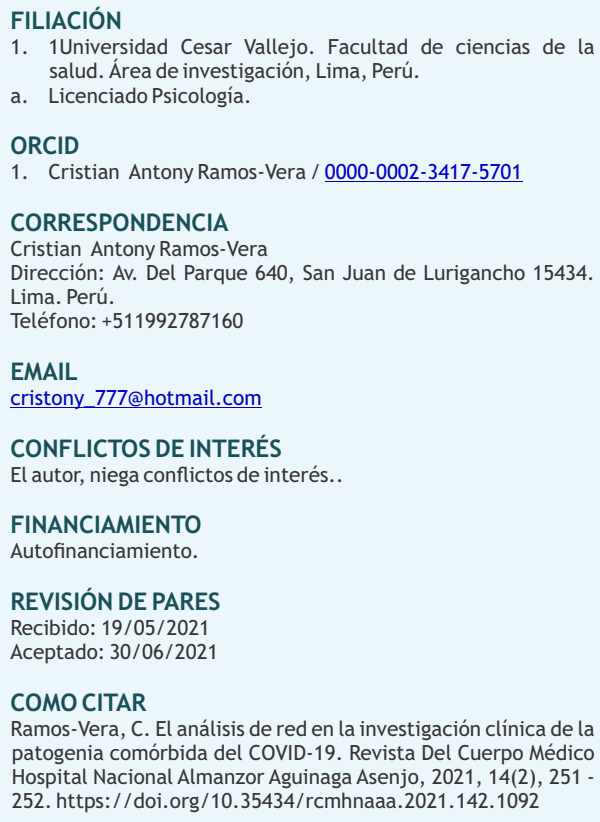

Ramos-Vera, C. El análisis de red en la investigación clínica de la patogenia comórbida del COVID-19. Revista Del Cuerpo Médico Hospital Nacional Almanzor Aguinaga Asenjo, 2021, 14(2), 251 252. https://doi.org/10.35434/rcmhnaaa.2021.142.1092 
salud comórbidos de la COVID-19 como la DM e HA y las posibles consecuencias neuropsiquiátricas.

Por ejemplo, un estudio brasileño evaluó las asociaciones en red de diversas enfermedades clínicas como la DM, osteoporosis, incontinencia urinaria, medidas antropométricas, fisiológicas y neuropsiquiátricas como la depresión y fragilidad ${ }^{(8)}$. Otra investigación de red evaluó las relaciones entre condiciones crónicas de salud como la DM, $\mathrm{HA}$, asma, problemas de espalda, migrañas, tabaquismo, así como la calidad de vida, estado de salud física y mental, y variables psicológicas como la ansiedad, depresión y psicosis ${ }^{(11)}$.

El modelo de red permite reportar los elementos centrales (altas medidas de centralidad de red) que refieren mayor implicancia clínica en la muestra de estudio pues permiten explicar los vínculos fisiopatológicos esenciales que exacerban el funcionamiento global de las diversas condiciones comórbidas al COVID-19 como la DM e HA ${ }^{(5,6)}$. Tales elementos centrales cercanos en la red afectan las interacciones de los componentes concomitantes, es decir, una mayor prevalencia de estos elementos aumenta la probabilidad de fortalecer las demás relaciones, y viceversa, la reducción de las medidas centrales permite plantear la hipótesis de que las conexiones del sistema se debiliten o desaparezcan dado que afianza el colapso estructural de la red, favorable al buen estado de salud. Esto es esencial en la planificación y desarrollo personalizado de intervenciones clínicas más eficaces a partir de los resultados obtenidos en el grupo de interés, que pueden diferir en el control glucémico, el nivel de presión arterial, el estado de salud mental, el tipo y nivel de medicación, así como la prevalencia de los síntomas consecuentes a la medicación por COVID-19, considerados como medidas en la red.

La inclusión de diversas covariables más comunes en la investigación clínica han orientado nuevas hipótesis mediante la evaluación simultánea de dos o más redes, por ejemplo, para comparar dos redes: con y sin el control de las covariables $^{(7)}$, o entre dos tiempos diferentes: antes y después de una intervención (pre-post test), donde la variación dinámica entre redes determine los efectos de un tratamiento mediante una menor medida de conexión entre los componentes centrales de la red. También es esencial para comprender la concurrencia interactiva de las medidas de red entre grupos según el riesgo del nivel de infección por COVID-19, la edad y el número de enfermedades comórbidas. En conclusión, el análisis de red ofrece una valiosa contribución metodológica y práctica en la investigación de ciencias de la salud para brindar una mayor explicación del funcionamiento dinámico (patrones asociativos de influencia mutua) de los factores de mayor vínculo patogénico al COVID19 como la DM. Esto permite fijar nuevas vías de investigación para desentrañar la complejidad de las enfermedades crónicas concomitantes asociadas con la edad ante el contexto pandémico como la hipótesis de la paradoja de la adiponectina que produce una mayor reacción inflamatoria y resistencia a la insulina ${ }^{(12)}$, mediante la inclusión de los niveles de la adiponectina, los patrones moleculares asociados al daño, puntuaciones de riesgo poligénico, las citocinas, factores metabólicos, biomarcadores inflamatorios de COVID-19, la DM, HA y otras medidas de manifestaciones comórbidas.

Los resultados de red permiten implementar una mejor atención integral y elaboración de estrategias terapéuticas más eficaces ante el impacto de la COVID-19 de etiología compleja y multifactorial desde el enfoque sistémico de modelos dinámicos de red de comorbilidad.

\section{REFERENCIAS BIBLIOGRÁFICAS}

1. Navarrete-Mejía P, Lizaraso-Soto F, Velasco-Guerrero J, Loro-Chero L. Diabetes mellitus e hipertensión arterial como factor de riesgo de mortalidad en pacientes con Covid-19. Rev Cuerpo Med. HNAAA, $2021 ; 13$ ( 4 ) : $361-365$. https: / / doi.org/10.35434/rcmhnaaa.2020.134.766

2. Kamyshnyi A, Krynytska I, Matskevych V, Marushchak M, Lushchak O. Arterial Hypertension as a Risk Comorbidity Associated with COVID-19 Pathology. Int J Hypertension, 2020.e 8019360. https: / / doi.org/10.1155/2020/8019360

3. Lim S, Bae JH, Kwon HS, Nauck MA. COVID-19 and diabetes mellitus: from pathophysiology to clinical management. Nat Rev Endocrinol. 2021;17(1):11-30. https://doi.org/10.1038/s41574-020-00435-4.

4. Muniangi-Muhitu H, Akalestou E, Salem V, Misra S, Oliver NS, Rutter GA. Covid-19 and Diabetes: A Complex Bidirectional Relationship. Front Endocrinol. 2020 ; 11 : e 582936 . https://doi.org/10.3389/fendo.2020.582936

5. Fried El, van Borkulo CD, Cramer AO, Boschloo L, Schoevers RA, Borsboom D. Mental disorders as networks of problems: a review of recent insights. Soc Psychiatry Psychiatr Epidemiol. 2017;52(1):1-10. https: / / doi.org/1007/s00127-016-1319-z

6. Ramos-Vera C. Las redes de relación estadística en la investigación de nutrición. Nutr Hosp. 2021. https: // doi.org/10.20960/nh.03522

7. Kappelmann N, Czamara D, Rost N, Moser S, Schmoll V, Trastulla L, et al. Polygenic risk for immuno-metabolic markers and specific depressive symptoms: A multi-sample network analysis study. medRxiv. 2021, https: / /doi.org/10.1101/2021.01.07.20248981

8. Leme DEDC, Alves EVDC, Fattori A. Relationships Between Social, Physical, and Psychological Factors in Older Persons: Frailty as an Outcome in Network Analysis. J Am Med Dir Assoc. 2020;21(9):13091315.e4. https://doi.org/10.1016/j.jamda.2020.02.005.

9. Ramos-Vera C, Baños-Chaparro, J, Ogundokun R. Network structure of depressive symptoms in Peruvian adults with arterial hy pertension. F1000Research. 2021; $10: 19$. https://doi.org/10.12688/f1000research.27422.2

10. Hilland E, Landrø NI, Kraft B, Tamnes CK, Fried El, Maglanoc LA, Jonassen R. Exploring the links between specific depression symptoms and brain structure: A network study. Psychiatry Clin Neurosci. 2020;74(3):220-221. https://doi.org/10.1111/pcn.12969.

11. Isvoranu AM, Abdin E, Chong SA, Vaingankar J, Boorsbom D, Subramaniam S. Extended network analysis: from psychopathology to chronic illness. BMC Psychiatry. 2021;21, 119. https: //doi.org/1186/s12888-021-03128-y

12. Ho G, Ali A, Takamatsu Y, Wada R, Masliah E, Hashimoto M. Diabetes, inflammation, and the adiponectin paradox: Therapeutic targets in SARS-CoV-2. Drug Discov Today. 2021: S1359-6446(21)00149-5. https://doi.org/10.1016/j.drudis.2021.03.013. 